\title{
Transfer of spin squeezing and particle entanglement between atoms and photons in coupled cavities via two-photon exchange
}

\author{
Ali Ü. C. Hardal and Özgür E. Müstecaplığ̆lu* \\ Department of Physics, Koç University, Sarıyer, Istanbul 34450, Turkey \\ ${ }^{*}$ Corresponding author: omustecap@ku.edu.tr
}

Received March 15, 2012; revised May 19, 2012; accepted May 20, 2012; posted May 22, 2012 (Doc. ID 164811); published June 27, 2012

\begin{abstract}
We examine transfer of particle entanglement and spin squeezing between atomic and photonic subsystems in optical cavities coupled by two-photon exchange. Each cavity contains a single atom, interacting with cavity photons with a two-photon cascade transition. Particle entanglement is characterized by evaluating optimal spin squeezing inequalities for the cases of initially separable and entangled two-photon states. It is found that particle entanglement is first generated among the photons in separate cavities and then transferred to the atoms. The underlying mechanism is recognized as an intercavity two-axis twisting spin squeezing interaction, induced by two-photon exchange, and its optimal combination with the intracavity atom-photon coupling. Relative effect of nonlocal two-photon exchange and local atom-photon interactions of cavity photons on the spin squeezing and entanglement transfer is pointed out. (C) 2012 Optical Society of America

OCIS codes: $\quad 270.4180,270.5580,270.5585$
\end{abstract}

\section{INTRODUCTION}

Recent investigations of quantum tunneling dynamics of two bosonic particles in photonic [1] and atomic [2] settings reveal their rich dynamical features not available to typical single particle tunneling situation. In the case of ultracold atoms in optical lattices, pairwise tunneling yields rich quantum phase diagrams [3]. It is proposed that two-boson tunneling can be realized by coupling molecular states to atomic ones. A similar scenario was considered before for spin squeezing $[4,5]$ and entanglement generation. Entanglement properties of two-photon exchange coupled cavities were analyzed in detail for the cases of two- and four-dimensional Hilbert space systems. These results could set the pathway toward massively correlated multiphoton nonlinear quantum optical systems [6,7], which are rapidly developing modern subjects nowadays. The motivation of interest to such systems is their promise in quantum switching, quantum communication and computation, and quantum phase transition applications.

Motivated by the link between the two-particle tunneling in atomic systems and spin squeezing, as well as its generation schemes based upon quantum state transfer between optical and atomic systems [믈 $\underline{13}$ ], our aim is to investigate a similar spin squeezing route to particle entanglement in a two-photon exchange coupled optical cavity system. Previous studies investigate entanglement in the whole atom-photon Hilbert space in two and four dimensions [1]. Our objective is to examine spin squeezing within photon and atom subsystems and to consider possible transfer of the particle entanglement between the two subsystems.

In this paper, we reveal the inherit two-axis twisting spin squeezing interaction nature of two-photon exchange interaction. The nonlocal interaction between the cavities induces spin squeezing in the photon subsystem. Induced spin squeezing in the photon subsystem is then transferred to the atomic subsystem by the local atom-photon coupling within each cavity. We consider the cases of initially entangled and nonentangled photons. Spin squeezing is witnessed by the optimal spin squeezing inequalities [14].

This paper is organized as follows. In Section 2 , we review the concept of spin squeezing and its relation to particle entanglement. In Section 3, we review the model system introduced in [1] and point out that the two-photon hopping interaction Hamiltonian is equivalent to the two-axis twisting Hamiltonian, which can generate squeezed spin states. In Section 4 , our results for the spin squeezing and particle entanglement transfer are presented in Subsections 4.A and 4.B for the initially entangled and nonentangled photonic subsystems, respectively. The role of quadrature squeezing for these transfers to occur is pointed out. In Subsection 4.C, the possible implementations of the model system are reviewed. We conclude in Section 5.

\section{SPIN SQUEEZING AND PARTICLE ENTANGLEMENT}

Spin squeezing [15] can be defined in terms of Heisenberg uncertainty. Such a definition is subjective to the choice of coordinate system. An alternative definition that takes into account quantum correlations between individual atomic spins is given by Kitagawa and Ueda [4] in the form

$$
\xi=\frac{\Delta J_{\perp}}{\sqrt{J / 2}}
$$

where $J_{\perp}$ is the angular momentum component in the direction of the unit vector $\vec{n}$ along which $\Delta \vec{n} \cdot \vec{J}$ is minimized and $J / 2$ is the total variance of each individual spin-1/2. If $\xi<1$, the system is said to be spin squeezed. 
Another spin squeezing criteria, which combines spin squeezing with particle entanglement, is given by [5]

$$
\xi_{e}^{2}=\frac{N\left(\Delta J_{n_{1}}\right)^{2}}{\left\langle J_{n_{2}}\right\rangle^{2}+\left\langle J_{n_{3}}\right\rangle^{2}} \geq 1,
$$

where $N$ is the number of particles in the system, $J_{n}=\vec{n} \cdot \vec{J}$, and the $\vec{n} s$ are mutually orthogonal unit vectors. The condition $\xi_{e}^{2}<1$ witnesses not only spin squeezing but also the particle entanglement. Particle entanglement happens in the first quantization and hence is fundamentally different than socalled mode entanglement that occurs in second quantization [16-18].

On the other hand, there exist states for which the spin squeezing parameter given in Eq. (2) cannot be used to analyze quantum entanglement [19]. Alternatively, one may use the following inequalities since it has been shown that [14] violation of any of them implies entanglement:

$$
\begin{gathered}
\left\langle J_{x}^{2}\right\rangle+\left\langle J_{y}^{2}\right\rangle+\left\langle J_{z}^{2}\right\rangle \leq \frac{N(N+2)}{4}, \\
\left(\Delta J_{x}\right)^{2}+\left(\Delta J_{y}\right)^{2}+\left(\Delta J_{z}\right)^{2} \geq \frac{N}{2}, \\
\left\langle J_{k}^{2}\right\rangle+\left\langle J_{l}^{2}\right\rangle-\frac{N}{2} \leq(N-1)\left(\Delta J_{m}\right)^{2}, \\
(N-1)\left[\left(\Delta J_{k}\right)^{2}+\left(\Delta J_{l}\right)^{2}\right] \geq\left\langle J_{m}^{2}\right\rangle+\frac{N(N-2)}{4},
\end{gathered}
$$

where $k, l, m$ take all the possible permutations of $x, y, z$. These inequalities are called optimal spin squeezing inequalities. Equation (3c) is equivalent to the Eq. (2) for a system of many particles that has a maximal spin in some direction [20], and they can be related to the positivity of concurrence [21]. Very recently, these inequalities generalized for qudits with arbitrary spin [22].

Entanglement is considered as a quantum information resource, and hence it is desirable to be able to transfer entanglement between distant nodes in a quantum network [요, $\underline{9}$. In Section 3 we will review the two-photon hopping model and argue that it can serve as an efficient tool for establishing particle entanglement between distant atoms via transfer of entanglement from a photon subsystem to an atom subsystem.

\section{MODEL}

We consider a system of two spatially separated optical cavities, each containing a single atom, that is interacting with the cavity field by two-photon transitions. The cavities are coupled to each other with two-photon exchange. The Hamiltonian of the system is written by $[\underline{1,23}-\underline{25}]$

$$
H=H^{(1)}-H_{0}^{(1)}+H^{(2)}-H_{0}^{(2)}+\hbar \zeta\left(a_{1}^{\dagger 2} a_{2}^{2}+a_{2}^{\dagger 2} a_{1}^{2}\right),
$$

where

$$
\begin{aligned}
H^{(i)}= & \hbar \omega a_{i}^{\dagger} a_{i}+\hbar \omega\left(\sigma_{e e}^{(i)}-\sigma_{g g}^{(i)}\right)+\hbar \mu \sigma_{e e}^{(i)} \\
& +\hbar \eta \sigma_{g g}^{(i)}+\hbar \lambda\left(\sigma_{e g}^{(i)} a_{i}^{2}+\sigma_{g e}^{(i)} a_{i}^{\dagger 2}\right),
\end{aligned}
$$

$$
H_{0}^{(i)}=\hbar \omega\left(a_{i}^{\dagger} a_{i}+\sigma_{e e}^{(i)}\right)-\sigma_{g g}^{(i)}+\left(E_{g}+E_{e}\right) 2
$$

with $E_{g}, E_{e}$ being the energies of the ground and exited states, respectively. The last term in Eq. (ㅁ) is the two-photon exchange interaction between the cavities, characterized by the hopping rate $\zeta$. The parameters $\mu, \eta$, and $\lambda$ are the free energies of the subsystems and the two-photon transition rate written in the convention of [26], $\sigma_{k l}^{(i)}=|k\rangle^{(i)(i)}\langle l|$ are the atomic transition operators, $k$ and $l$ denote either ground $(g)$ or exited $(e)$ states of the atom, and $i=1,2$ labels the cavities. Throughout this paper, the hopping rate $\zeta$, time $t$, and the eigenfrequencies $\omega_{i} s$ will be scaled by the two-photon transition rate $\lambda$ in numerical calculations. Therefore, in all figures, $\zeta \leq 1$ corresponds to the local interaction dominant regimes, while $\zeta>1$ corresponds to the hopping dominant regimes.

The spin components for a system of two-level atoms are defined by [12]

$$
\begin{aligned}
S_{x} & =\frac{1}{2}\left(\sigma_{e g}+\sigma_{g e}\right), \\
S_{y} & =\frac{-i}{2}\left(\sigma_{e g}-\sigma_{g e}\right), \\
S_{z} & =\frac{1}{2}\left(\sigma_{e e}-\sigma_{g g}\right) .
\end{aligned}
$$

Since we are interested in two coupled cavities, each having one atom, we define the spin components of the atoms for this configuration as follows:

$$
\begin{aligned}
& S_{x}=S_{x}^{(1)} \otimes 1^{(2)}+1^{(1)} \otimes S_{x}^{(2)}, \\
& S_{y}=S_{y}^{(1)} \otimes 1^{(2)}+1^{(1)} \otimes S_{y}^{(2)}, \\
& S_{z}=S_{z}^{(1)} \otimes 1^{(2)}+1^{(1)} \otimes S_{z}^{(2)},
\end{aligned}
$$

where the superscripts (1) and (2) are labeling the first and second cavities, respectively.

Likewise, we can consider pseudo-spin operators for the coupled cavity field such that

$$
\begin{aligned}
& L_{x} \equiv \frac{1}{2}\left(a_{1}^{\dagger} a_{2}+a_{2}^{\dagger} a_{1}\right), \\
& L_{y} \equiv \frac{-i}{2}\left(a_{1}^{\dagger} a_{2}-a_{2}^{\dagger} a_{1}\right), \\
& L_{z} \equiv \frac{1}{2}\left(a_{1}^{\dagger} a_{1}-a_{2}^{\dagger} a_{2}\right) .
\end{aligned}
$$

They satisfy the SU(2) spin algebra $\left[L_{\alpha}, L_{\beta}\right]=\epsilon^{\alpha \beta \gamma} L_{\gamma}$. Here $\alpha, \beta, \gamma \in\{x, y, z\}$ and $\epsilon^{\alpha \beta \gamma}$ is the Levi-Civita density. In terms of $L_{+} \equiv L_{x}+i L_{y}=a_{1}^{\dagger} a_{2}$ and $L_{-} \equiv L_{x}-i L_{y}=a_{2}^{\dagger} a_{1}$, it is straightforward to rewrite two-photon hopping interaction Hamiltonian $H_{p}=\hbar \zeta\left(a_{1}^{\dagger 2} a_{2}^{2}+a_{2}^{\dagger 2} a_{1}^{2}\right)$ as

$$
H_{p}=\hbar \zeta\left(L_{+}^{2}+L_{-}^{2}\right)=2 \hbar \zeta\left(L_{x}^{2}-L_{y}^{2}\right) .
$$

This is nothing but the two-axis twisting Hamiltonian, which squeezes the spin states by twisting them about the two axes [4]. Clearly neither spin squeezing nor associated particle entanglement can be achieved via one photon hopping in coupled cavity systems, as the coupling term would be a mere rotation operation generated by $L_{x}$, though it would produce mode entanglement. 
It should be noted that, in collective spin systems, i.e., systems in which all spins mutually interact, Eq. (10) is known as the Lipkin-Meshkov-Glick (LMG) Hamiltonian, whose entanglement dynamics and exact properties in the thermodynamical limit have been carefully analyzed in [27-29]. Alternatively, the two-photon hopping can also be seen as a special case of more general nonlinear quantum optical Karassiov-Klimov models [30]. Following the Lie-algebraic approach, a twophoton hopping term can be recognized by a Jordan-Schwinger map as the difference of raising and lowering operators of the Higgs algebra [31]. In the view of the background field method in the Fokker-Planck formalism [32], we then expect that fluctuations of (Higgs or deformed) effective photon spin operators drive fluctuations of collective atomic spin operators coupled to two-photon transition operators. Fluctuations of two-photon transition operators can be further linked to the quadrature variances. We would then intuitively expect that reduction of spin noise from the photon subsystem could be transferred to the atomic subsystem through reduction of quadrature noise. We shall numerically investigate these intuitive expectations for some initial cases in the Section 4 .

\section{SQUEEZING AND ENTANGLEMENT TRANSFER}

In this section we investigate the transfer of spin squeezing and particle entanglement between distant cavities. To do that, we shall obtain the optimal spin squeezing inequalities for the atomic and photonic systems separately, analyze their variations in time, and compare them with each other.

For a given initial state $|\psi(0)\rangle$, restricted to two-photon manifold, the time-dependent state vector $|\psi(t)\rangle$ may be written as

$$
\begin{aligned}
|\psi(t)\rangle= & A(t)|g, 2\rangle^{1}|g, 0\rangle^{2}+B(t)|g, 0\rangle^{1}|g, 2\rangle^{2} \\
& +C(t)|g, 0\rangle^{1}|e, 0\rangle^{2}+D(t)|e, 0\rangle^{1}|g, 0\rangle^{2} .
\end{aligned}
$$

The general solution of the time-dependent Schrödinger equation $i \hbar \partial_{t}|\psi(t)\rangle=H|\psi(t)\rangle$ in the two-dimensional submanifold of the Hilbert space is [1]

$$
\begin{aligned}
|\psi(t)\rangle= & \left(A_{1} e^{-i \omega_{1} t}+A_{2} e^{-i \omega_{2} t}\right)\left|\phi_{1}\right\rangle+\left(A_{3} e^{-i \omega_{3} t}+A_{4} e^{-i \omega_{4} t}\right)\left|\phi_{2}\right\rangle \\
& +\left(\alpha_{1} A_{1} e^{-i \omega_{1} t}+\alpha_{2} A_{2} e^{-i \omega_{2} t}\right)\left|\phi_{3}\right\rangle \\
& \times\left(\alpha_{3} A_{3} e^{-i \omega_{3} t}+\alpha_{4} A_{4} e^{-i \omega_{4} t}\right)\left|\phi_{4}\right\rangle
\end{aligned}
$$

where $\alpha_{i}=\sqrt{2} / 2\left(\omega_{i}-0.5\right), \omega_{i} s$ are the eigenfrequencies described explicitly in [1] as well as $A_{i} s$ and

$$
\begin{aligned}
& \left|\phi_{1}\right\rangle=\frac{1}{\sqrt{2}}\left(|g, 2\rangle^{1}|g, 0\rangle^{2}+|g, 0\rangle^{1}|g, 2\rangle^{2}\right), \\
& \left|\phi_{2}\right\rangle=\frac{1}{\sqrt{2}}\left(|g, 2\rangle^{1}|g, 0\rangle^{2}-|g, 0\rangle^{1}|g, 2\rangle^{2}\right), \\
& \left|\phi_{3}\right\rangle=\frac{1}{\sqrt{2}}\left(|e, 0\rangle^{1}|g, 0\rangle^{2}+|g, 0\rangle^{1}|e, 0\rangle^{2}\right), \\
& \left|\phi_{4}\right\rangle=\frac{1}{\sqrt{2}}\left(|e, 0\rangle^{1}|g, 0\rangle^{2}-|g, 0\rangle^{1}|e, 0\rangle^{2}\right) .
\end{aligned}
$$

The states in Eqs. (13) are mutually orthogonal and entangled. Since this solution allows for Rabi oscillations, one can transfer entanglement between the symmetric $\left(\left|\phi_{1}\right\rangle\right.$ and $\left.\left|\phi_{3}\right\rangle\right)$ and antisymmetric $\left(\left|\phi_{2}\right\rangle\right.$ and $\left.\left|\phi_{4}\right\rangle\right)$ states separately [1].

\section{A. Initially Entangled Photons}

Let us first start with a state that is initially entangled:

$$
|\psi(0)\rangle=\frac{1}{\sqrt{2}}\left(|g, 2\rangle^{1}|g, 0\rangle^{2}+|g, 0\rangle^{1}|g, 2\rangle^{2}\right)
$$

Then, at time $t$, we have

$|\psi(t)\rangle=A_{1}\left(e^{-i \omega_{1} t}+\alpha_{1}^{2} e^{-i \omega_{2} t}\right)\left|\phi_{1}\right\rangle+\alpha_{1} A_{1}\left(e^{-i \omega_{1} t}-e^{-i \omega_{2} t}\right)\left|\phi_{3}\right\rangle$

with $A_{1}+\alpha_{1}^{2} A_{1}=1$. For the atom-photon system, the density operator, $\rho_{\text {ap }}^{12}(t)$, becomes

$$
\begin{aligned}
\rho_{\mathrm{ap}}^{12}(t)= & |A|^{2}\left|\phi_{1}\right\rangle\left\langle\phi_{1}\left|+A B^{*}\right| \phi_{1}\right\rangle\left\langle\phi_{3}\right| \\
& +B A^{*}\left|\phi_{3}\right\rangle\left\langle\left.\phi_{1}|+| B\right|^{2} \mid \phi_{3}\right\rangle\left\langle\phi_{3}\right|,
\end{aligned}
$$

where

$$
\begin{aligned}
& |A|^{2}=A_{1}^{2}\left[1+2 \alpha_{1}^{2} \cos \left(\delta_{12} t\right)+\alpha_{1}^{4}\right], \\
& A B^{*}=\alpha_{1} A_{1}^{2}\left[1-\alpha_{1}^{2}+\alpha_{1}^{2} e^{i \delta_{12} t}-e^{-i \delta_{12} t}\right], \\
& |B|^{2}=2 \alpha_{1}^{2} A_{1}^{2}\left[1-\cos \left(\delta_{12} t\right)\right],
\end{aligned}
$$

and $\delta_{12}=\omega_{1}-\omega_{2}$. To obtain reduced density operators $\rho_{a}^{12}(t)$ and $\rho_{p}^{12}(t)$ for atomic and photonic systems, respectively, we take partial traces as

$$
\begin{aligned}
\rho_{a}^{12}(t)= & \operatorname{Tr}_{p} \rho_{\mathrm{ap}}^{12}(t)=|A|^{2}|g\rangle^{11}\langle g|\otimes| g\rangle^{22}\langle g| \\
& +\frac{|B|^{2}}{2}\left(|e\rangle^{11}\langle e|\otimes| g\rangle^{22}\langle g|+| e\rangle^{11}\langle g|\otimes| g\rangle^{22}\langle e|\right. \\
& \left.+|g\rangle^{11}\langle e|\otimes| e\rangle^{22}\langle g|+| g\rangle^{11}\langle g|\otimes| e\rangle^{22}\langle e|\right), \\
\rho_{p}^{12}(t) & =\operatorname{Tr}_{a} \rho_{\mathrm{ap}}^{12}(t)=\frac{|A|^{2}}{2}(|2,0\rangle\langle 2,0|+| 2,0\rangle\langle 0,2| \\
& +|0,2\rangle\langle 2,0|+| 0,2\rangle\langle 0,2|)+|B|^{2}|0,0\rangle\langle 0,0| .
\end{aligned}
$$

Equation (3d) gives the following inequalities for atomic and photonic subsystems, respectively:

$$
\begin{aligned}
& \text { Ineq }_{a} \equiv 4-5|A|^{2} \leq 0, \\
& \text { Ineq }_{p} \equiv|A|^{2} \leq 0,
\end{aligned}
$$

where we take $k=x, l=z, m=y$ in Eq. (20) and $k=z$, $l=y, m=x$ in Eq. (21). Violation of these inequalities implies spin squeezing (and particle entanglement) in the corresponding systems, and, as can be easily seen from the Fig. 1, indeed this is the case. Initially, we have nonentangled atoms and entangled photons. As time goes on, the atomic system becomes entangled, which is induced by the photons; i.e., 
we have entanglement and spin squeezing transfer. Transfers occur periodically in the local-interaction regime $(\zeta<1)$. In the hopping dominant regime, atoms are not entangled or spin squeezed, while the photons are. As should be expected, almost pure two-photon entanglement regimes occur more frequently and in broader intervals with the increase of the twophoton hopping rate.

\section{B. Initially Nonentangled State}

We next consider initially a nonentangled state of the form

$$
|\psi(0)\rangle=|g, 2\rangle^{1}|g, 0\rangle^{2},
$$

which evolves in time as

$$
\begin{aligned}
|\psi(t)\rangle= & A_{1}\left(e^{-i \omega_{1} t}+\alpha_{1}^{2} e^{-i \omega_{2} t}\right)\left|\phi_{1}\right\rangle \\
& +\alpha_{1} A_{1}\left(e^{-i \omega_{1} t}-e^{-i \omega_{2} t}\right)\left|\phi_{3}\right\rangle \\
& +A_{3}\left(e^{-i \omega_{3} t}+\alpha_{3}^{2} e^{-i \omega_{4} t}\right)\left|\phi_{2}\right\rangle \\
& +\alpha_{3} A_{3}\left(e^{-i \omega_{3} t}-e^{-i \omega_{4} t}\right)\left|\phi_{4}\right\rangle .
\end{aligned}
$$

The density operator, $\rho_{\mathrm{ap}}^{12}$, for this case then reads

$$
\begin{aligned}
\rho_{\mathrm{ap}}^{12}(t)= & |A|^{2}\left|\phi_{1}\right\rangle\left\langle\phi_{1}\left|+A B^{*}\right| \phi_{1}\right\rangle\left\langle\phi_{3}\left|+A C^{*}\right| \phi_{1}\right\rangle\left\langle\phi_{2}\right| \\
& +A D^{*}\left|\phi_{1}\right\rangle\left\langle\phi_{4}\left|+B A^{*}\right| \phi_{3}\right\rangle\left\langle\left.\phi_{1}|+| B\right|^{2} \mid \phi_{3}\right\rangle\left\langle\phi_{3}\right| \\
& +B C^{*}\left|\phi_{3}\right\rangle\left\langle\phi_{2}\left|+B D^{*}\right| \phi_{3}\right\rangle\left\langle\phi_{4}\left|+C A^{*}\right| \phi_{2}\right\rangle\left\langle\phi_{1}\right| \\
& +C B^{*}\left|\phi_{2}\right\rangle\left\langle\left.\phi_{3}|+| C\right|^{2} \mid \phi_{2}\right\rangle\left\langle\phi_{2}\left|+C D^{*}\right| \phi_{2}\right\rangle\left\langle\phi_{4}\right| \\
& +D A^{*}\left|\phi_{4}\right\rangle\left\langle\phi_{1}\left|+D B^{*}\right| \phi_{4}\right\rangle\left\langle\phi_{3}\left|+D C^{*}\right| \phi_{4}\right\rangle\left\langle\phi_{2}\right| \\
& +|D|^{2}\left|\phi_{4}\right\rangle\left\langle\phi_{4}\right|,
\end{aligned}
$$

where

$$
\begin{aligned}
A C^{*}= & A_{1} A_{3}\left[e^{i\left(\omega_{3}-\omega_{1}\right) t}+\alpha_{3}^{2} e^{i\left(\omega_{4}-\omega_{1}\right) t}\right. \\
& \left.+\alpha_{1}^{2} e^{i\left(\omega_{3}-\omega_{2}\right) t}+\alpha_{1}^{2} \alpha_{3}^{2} e^{i\left(\omega_{4}-\omega_{2}\right) t}\right],
\end{aligned}
$$
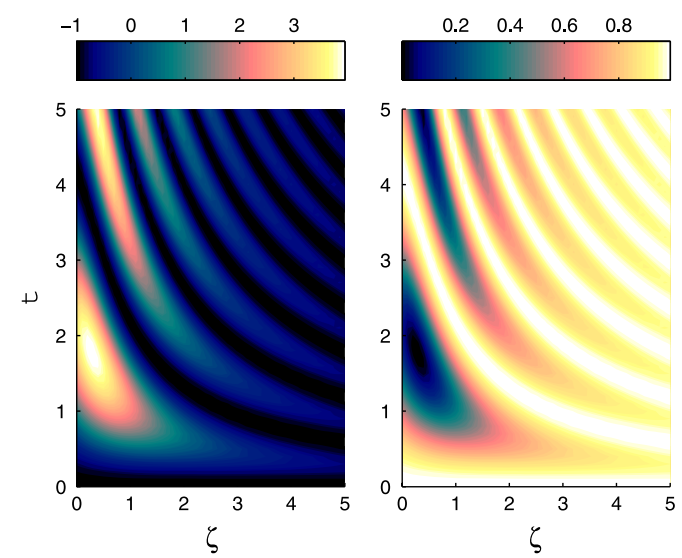

Fig. 1. (Color online) Variation of spin squeezing in (left) atomic and (right) photonic subsystems with respect to time and hopping constant. $\zeta$ is scaled by $\lambda$, and $\zeta \leq 1$ corresponds to the local-interaction dominant regimes, while $\zeta>1$ corresponds to the hopping dominant regimes. Spin squeezing is characterized positive values in both figures. At $t=0$ Ineq $_{a}=-1$ and Ineq $_{p}=1$. All the parameters plotted are dimensionless as explained in the text.

$$
\begin{aligned}
A D^{*}= & \alpha_{3} A_{1} A_{3}\left[e^{i\left(\omega_{3}-\omega_{1}\right) t}-e^{i\left(\omega_{4}-\omega_{1}\right) t}\right. \\
& \left.+\alpha_{1}^{2}\left(e^{i\left(\omega_{3}-\omega_{2}\right) t}-e^{i\left(\omega_{4}-\omega_{2}\right) t}\right)\right] \\
B C^{*}= & \alpha_{1} A_{1} A_{3}\left[e^{i\left(\omega_{3}-\omega_{1}\right) t}-e^{i\left(\omega_{3}-\omega_{2}\right) t}\right. \\
& \left.+\alpha_{3}^{2}\left(e^{i\left(\omega_{4}-\omega_{1}\right) t}-e^{i\left(\omega_{4}-\omega_{3}\right) t}\right)\right]
\end{aligned}
$$

$$
\begin{aligned}
B D^{*}= & \alpha_{1} \alpha_{3} A_{1} A_{3}\left[e^{i\left(\omega_{3}-\omega_{1}\right) t}+e^{i\left(\omega_{4}-\omega_{2}\right) t}\right. \\
& \left.-e^{i\left(\omega_{4}-\omega_{1}\right) t}-e^{i\left(\omega_{3}-\omega_{2}\right) t}\right]
\end{aligned}
$$

$$
C D^{*}=\alpha_{3} A_{3}^{2}\left[1-e^{i\left(\omega_{4}-\omega_{3}\right) t}+\alpha_{3}^{2}\left(e^{i\left(\omega_{3}-\omega_{4}\right) t}-1\right)\right],
$$

$$
\begin{gathered}
|C|^{2}=A_{3}^{2}\left[1+2 \alpha_{3}^{2} \cos \left[\left(\omega_{3}-\omega_{4}\right) t\right]+\alpha_{3}^{4}\right], \\
|D|^{2}=2 \alpha_{3}^{2} A_{3}^{2}\left[1-\cos \left[\left(\omega_{3}-\omega_{4}\right) t\right]\right],
\end{gathered}
$$

with $A_{1}\left(1+\alpha_{1}^{2}\right)=A_{3}\left(1+\alpha_{3}^{2}\right)=1 / \sqrt{2}$, and $|A|^{2},|B|^{2}, A B^{*}$ are given in Eqs. (17). The reduced density operators are given by

$$
\begin{aligned}
\rho_{a}^{12}(t)= & \left(|A|^{2}+|C|^{2}\right)|g\rangle^{11}\langle g|\otimes| g\rangle^{22}\langle g| \\
& +\frac{1}{2}\left[\left(|B|^{2}+|D|^{2}+B D^{*}+D B^{*}\right)|e\rangle^{11}\langle e|\otimes| g\rangle^{22}\langle g|\right. \\
& +\left(|B|^{2}-|D|^{2}-B D^{*}+D B^{*}\right)|e\rangle^{11}\langle g|\otimes| g\rangle^{22}\langle e| \\
& +\left(|B|^{2}-|D|^{2}+B D^{*}-D B^{*}\right)|g\rangle^{11}\langle e|\bigotimes| e\rangle^{22}\langle g| \\
+ & \left.\left(|B|^{2}+|D|^{2}-B D^{*}-D B^{*}\right)|g\rangle^{11}\langle g|\bigotimes| e\rangle^{22}\langle e|\right], \\
& \rho_{p}^{12}(t)= \\
& +\left(\left(|A|^{2}+A C^{*}+C A^{*}\right)|2,0\rangle\langle 2,0|\right. \\
& +\left(|A|^{2}-A C^{*}+C A^{*}\right)|2,0\rangle\langle 0,2| \\
& +\left(|A|^{2}-A C^{*}-C A^{*}\right)|0,2\rangle\langle 2,0| \\
& \left.+\left(2|B|^{2}+|D|^{2}\right)|0,0\rangle\langle 0,0\rangle\langle 0,2|\right]
\end{aligned}
$$

Then, Eq. (3d) gives

$$
\text { Ineq }_{a} \equiv 3|B|^{2}-|D|^{2}-2\left(|B|^{2}+|D|^{2}\right)^{2} \geq 0
$$

for the atomic system, and Eq. (3b) gives

$$
\text { Ineq }_{p} \equiv 2|A|^{2}-1 \geq 0,
$$

for the photonic system, where we take $k=x, l=z$, and $m=y$. As in the initially entangled case, violation of these inequalities implies spin squeezing (and particle entanglement) in the corresponding systems. Figure 2 shows that initially unentangled photons quickly get entangled, and this is transferred to the atomic subsystem similar to Fig. 1. Figure 2 verifies the periodical nature of the transfers by anchoring a hopping constant and observing the time evolutions. In terms of particle entanglement, then the atomic system becomes more correlated at the expense of the correlation of the field particles and vice versa.

Dynamics of correlations can be further interpreted by investigating the quadratures of the photonic subsystems. Local 

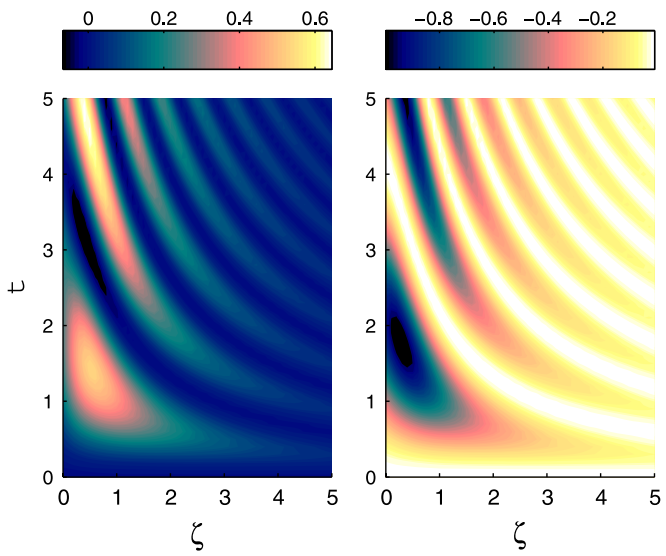

Fig. 2. (Color online) Variations of the spin squeezing in (left) atomic and (right) photonic subsystems with respect to time and hopping constant. Both inequalities are violated; therefore, we have both spin squeezed and entangled systems. Spin squeezing is characterized by negative values in both figures. At $t=0$, both inequalities are equal to zero. All the parameters plotted are dimensionless as explained in the text.

interaction of the photon-atom subsystems are of two-photon transition types, and thus we expect quadrature correlations to directly influence the atomic spin noise.

The quadrature operators for the field are defined as

$$
\begin{aligned}
& X_{1}=\frac{1}{2}\left(a^{\dagger}+a\right), \\
& X_{2}=\frac{i}{2}\left(a^{\dagger}-a\right) .
\end{aligned}
$$

Using the reduced density operators acting on the photonic subsystems, it can be readily shown that

$$
\begin{gathered}
\left(\Delta X_{1}\right)^{2}=\left(\Delta X_{2}\right)^{2}=\frac{1}{4}+\frac{1}{2}|A|^{2}, \\
\left(\Delta X_{1}\right)^{2}=\left(\Delta X_{2}\right)^{2}=\frac{7}{8}|A|^{2}+\frac{1}{4}\left(A C^{*}+C A^{*}+|D|^{2}\right)+\frac{1}{2}|B|^{2},
\end{gathered}
$$

for initially entangled and initially nonentangled states, respectively. As we see from Fig. 3, for the initially entangled state (left) we have ideally squeezed states at darker regions where the product of the variances of the quadratures is equal to 0.25 [since $\left(\Delta X_{1}\right)^{2}=\left(\Delta X_{2}\right)^{2}$ ]. The behavior of the quadratures is very similar to that of spin squeezing inequalities for the initially entangled field (see Fig. 1).

In the case that the state is initially nonentangled, the situation is even more revealing. We see from Fig. 3 (right) that initially there is no quadrature squeezing. But at later times we observe such correlations; especially at the darker regions we have $\left(\Delta X_{1}\right)^{2}=\left(\Delta X_{2}\right)^{2} \leq 1 / 4$. Thus, in the light of above observations about quadratures, we conclude that (i) a photon subsystem corresponds to effectively a spin system that is, under two-axis twisting interaction, squeezed and that (ii) particle entangled atoms do not directly interact with this effective spin system but couple to the field by two-photon transitions that can be linked to quadrature squeezing. (iii) Sharing the
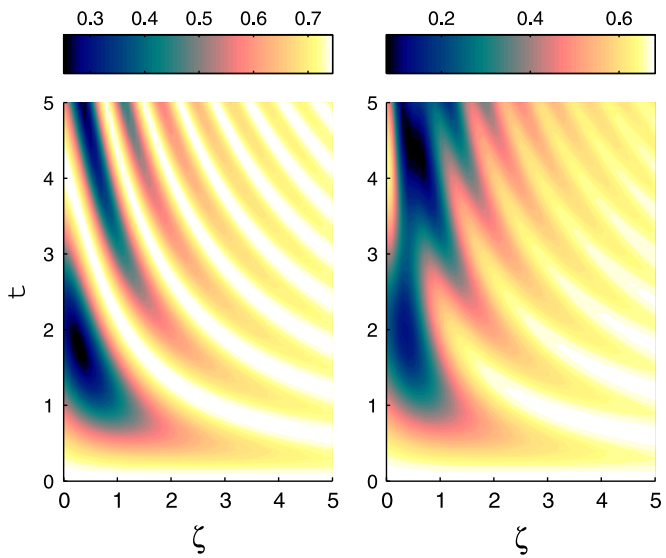

Fig. 3. (Color online) Variation of the variances of the quadratures for (left) initially entangled state and (right) initially nonentangled state with respect to time and hopping constant. Quadratures are squeezed for values $\leq 0.25$. At $t=0,\left(\Delta X_{1}\right)^{2}=\left(\Delta X_{2}\right)^{2}=0.75$ for initially entangled state and $\left(\Delta X_{1}\right)^{2}=\left(\Delta X_{2}\right)^{2}=0.6875$ for initially nonentangled state. All the parameters plotted are dimensionless as explained in the text.

quadrature squeezed particle entangled photons, the atoms build up sufficient noise reduction in particular directions to become spin squeezed and hence get particle entangled.

\section{Physical Implementation}

Before concluding, we briefly review the proposals for the physical realization of the model that we have examined throughout this paper. In Fig. $\underline{4}$, we illustrate a general scheme of possible realizations of the model $[\underline{1}, \underline{26}, 33]$. The scheme depicts two identical coupled resonators, with cavity mode frequencies $\omega$. Each resonator contains a three-level atom that interacts with the cavity mode in cascade formation. The detuning is given by $\Delta=E_{1}-E_{2}+\hbar \omega=E_{3}-E_{2}-\hbar \omega$, where $E_{1,2,3}$ are the energies of the corresponding levels of the atoms [26]. One of the three levels, the state $|2\rangle$, is eliminated via a unitary transformation $[\underline{1}, \underline{23}-25]$. The quantum states $|1\rangle$ and $|3\rangle$ correspond respectively to the $|g\rangle$ and $|e\rangle$ we have used in

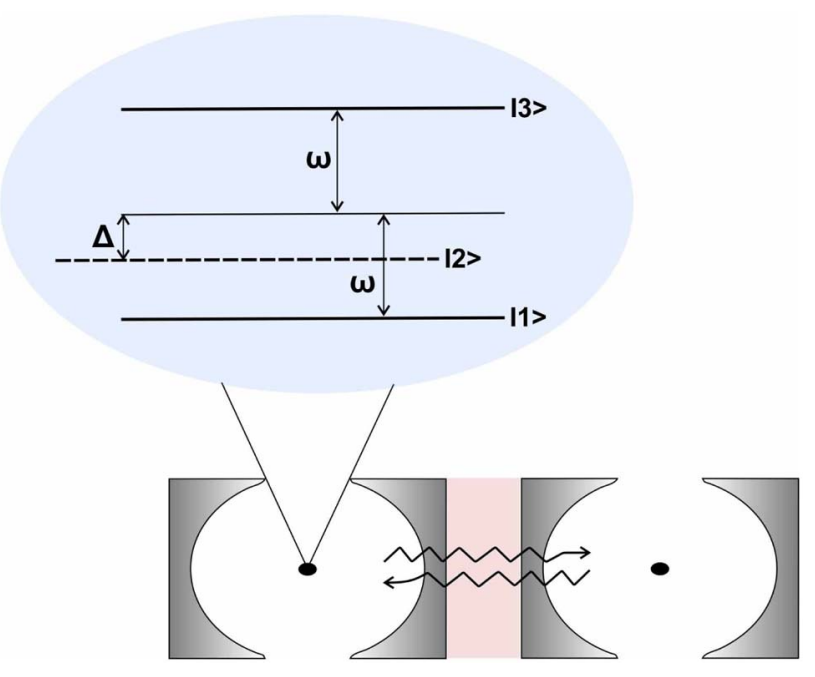

Fig. 4. (Color online) Two identical cavities, with cavity frequencies $\omega$, coupled via two-photon exchange, which is governed by a nonlinear Kerr medium. Each cavity contains a three-level atom in cascade formation. 
the earlier sections in the text. The cavities are coupled via a Kerr-type nonlinear dielectric medium that allows for twophoton interactions. It is proposed that this scheme can be implemented with superconducting circuit quantum electrodynamics (QED) technology [33]. The proposal is based upon an extension of single photon scattering and hopping in a system of two coupled superconducting transmission line resonators (TLRs). Two-photon hopping is suggested to be realized by using a nonlinear intermediate medium to couple the resonators [33]. The nonlinear dielectric causes Kerr-type interactions [7] among the TLR photons. It is further proposed that [1] an effective two-level system with two-photon transitions coupled to the resonators can be realized by using transmon qubits coupled to the resonators at their microwave field antinodes [34]. Circuit QED architectures with multilevel solid-state qubits are considered as promising systems to perform quantum information transfer operations [35]. Quantum state transfer in similar setups, but without twophoton hopping, in circuit QED has experimentally demonstrated [36].

The model parameters $\mu, \eta$, and $\lambda$ are all of the order of the qubit-resonator coupling, as can be seen from their expressions given in Appendix A. $\lambda$ determines the time and energy scales that are used in reporting our results. The critical condition to observe the reported transfer of spin squeezing is then to make sure the qubit-resonator coupling is sufficiently strong relative to the decoherence rate due to solid-state qubit dephasing and cavity losses. This seems to be plausible considering the recent progress toward ultrastrong coupling [37] and deep strong coupling [38] regimes of circuit QED. The hopping rate $\zeta$ is used in the text as a control parameter in our calculations, so we present our results in terms of the relative strength of $g$ and $\zeta$. In other words, spin squeezing is discussed as a function of relative strength of local on-cavity interaction of the qubit with respect to nonlocal intercavity coupling.

The initial states considered in the text can be realized within such circuit QED setups, similar to that in [39], where the authors have proposed a system composed of a superconducting phase qubit coupled to a microwave resonator, that may be used for the controlled generation of multiphoton Fock states. Finally the observation of the squeezing can be performed by using similar methods developed for second-order correlation function measurement [40].

\section{CONCLUSION}

Summarizing, we examine a system of two cavities containing single atoms coupled to the cavity field by two-photon transitions. The cavities are coupled by two-photon hopping interaction. We write the coupling as a two-axis twisting model of spin squeezing, which is also equivalent to the LMG model. We investigated spin squeezing and particle entanglement in atom and photon subsystems. We consider two cases: one of them is initially entangled and the other is initially nonentangled. In both cases, we analytically and numerically determine that both spin squeezing and entanglement are transferred from the photonic subsystem to the atomic subsystem and vice versa. We briefly argued that coupling of the atomic collective spin variable and the effective photonic spin is not a spin-spin interaction but of the form in which the quadrature correlations seem to be playing a crucial role. We numerically verified that the transfer of spin squeezing between photons and atoms could be mediated by quadrature squeezing.

\section{APPENDIX A}

We give expressions for the parameters $\mu, \eta$, and $\lambda$, which specify the effective free energies of the subsystems and the effective two-photon transition rate in an effective two-level system, respectively. They are generated by a unitary transformation method applied to a three-level atom in cascade formation [26]. Such a relation adds the model Hamiltonian, given in $\overline{\mathrm{Eq}}$. 4, intensity-dependent Stark-shift terms [26] so that they are depending on the photon number through the parameter $N$ defined below. Following the convention of the [26], these parameters are given by

$$
\begin{aligned}
& \hbar \lambda=\frac{g_{12} g_{23}\left\{\left[\Delta^{2}+4 \hbar^{2}\left(\tilde{g}_{12}^{2}+\tilde{g}_{23}^{2}\right)\right]^{1 / 2}-\Delta\right\}}{2\left(\tilde{g}_{12}^{2}+\tilde{g}_{23}^{2}\right)} \\
& \hbar \mu=\frac{\Delta \tilde{g}_{12}^{2}}{2\left(\tilde{g}_{12}^{2}+\tilde{g}_{23}^{2}\right)}+\frac{\left(\tilde{g}_{12}^{2}+2 \tilde{g}_{23}^{2}\right)\left[\Delta^{2}+4 \hbar^{2}\left(\tilde{g}_{12}^{2}+\tilde{g}_{23}^{2}\right)\right]^{1 / 2}}{2\left(\tilde{g}_{12}^{2}+\tilde{g}_{23}^{2}\right)} \\
& \hbar \eta=\frac{\Delta \tilde{g}_{23}^{2}}{2\left(\tilde{g}_{12}^{2}+\tilde{g}_{23}^{2}\right)}+\frac{\left(2 \tilde{g}_{12}^{2}+\tilde{g}_{23}^{2}\right)\left[\Delta^{2}+4 \hbar^{2}\left(\tilde{g}_{12}^{2}+\tilde{g}_{23}^{2}\right)\right]^{1 / 2}}{2\left(\tilde{g}_{12}^{2}+\tilde{g}_{23}^{2}\right)}
\end{aligned}
$$

where $g_{12}$ and $g_{23}$ are the coupling constants for the cavity and the corresponding transitions of the atomic levels (see Fig. 4), $\tilde{g}_{12}^{2}=g_{12}^{2} N, \tilde{g}_{23}^{2}=g_{23}^{2}(N-1)$ with $N=\left\langle a^{\dagger} a\right\rangle+\left\langle\sigma_{33}\right\rangle-\left\langle\sigma_{11}\right\rangle+$ 1 is a constant of motion $[1,26]$, and $\Delta$ is the detuning. Here, for the clarity of equations, we did not label the parameters to distinguish cavities since they are taken to be identical.

\section{ACKNOWLEDGMENTS}

We gratefully acknowledge inspiring comments by J. Vidal. Ö. E. M. acknowledges support by National Science and Technology Foundation of Turkey (TÜBİTAK) under project nos. 109T267 and 111T285.

\section{REFERENCES}

1. M. Alexanian, "Two-photon exchange between two three-level atoms in separate cavities," Phys. Rev. A 83, 023814 (2011).

2. S. Longhi, "Optical realization of two-boson tunneling dynamics," Phys. Rev. A 83, 43835 (2011).

3. X.-F. Zhou, Y.-S. Zhang, and G.-C. Guo, "Pair tunneling of bosonic atoms in an optical lattice," Phys. Rev. A 80, 013605 (2009).

4. M. Kitagawa and M. Ueda, "Squeezed spin states," Phys. Rev. A 47, 5138-5143 (1993).

5. A. Sørensen, L. M. Duan, J. I. Cirac, and P. Zoller, "Many-particle entanglement with Bose-Einstein condensates," Nature 409, 63-66 (2001)

6. M. Hillery, "An introduction to the quantum theory of nonlinear optics," Acta Phys. Slovaca 59, 1-80 (2009).

7. F. DellaAnno, S. De Siena, and F. Illuminati, "Multiphoton quantum optics and quantum state engineering," Phys. Rep. 428, 53-168 (2006).

8. A. Biswas and G. S. Agarwal, "Transfer of an unknown quantum state, quantum networks, and memory," Phys. Rev. A 70, 022323 (2004).

9. J. I. Cirac, P. Zoller, H. J. Kimble, and H. Mabuchi, "Quantum state transfer and entanglement distribution among distant 
nodes in a quantum network," Phys. Rev. Lett. 78, 3221-3224 (1997).

10. L. Vernac, M. Pinard, and E. Giacobino, "Quantum state transfer from light beams to atomic ensembles," Eur. Phys. J. D 17, 125-136 (2001).

11. J. Hald, J. L. Sørensen, C. Schori, and E. S. Polzik, "Spin squeezed atoms: a macroscopic entangled ensemble created by light," Phys. Rev. Lett. 83, 1319-1322 (1999).

12. A. Kuzmich, K. Mølmer, and E. S. Polzik, "Spin squeezing in an ensemble of atoms illuminated with squeezed light," Phys. Rev. Lett. 79, 4782-4785 (1997).

13. A. Banerjee, "Generation of atomic-squeezed states in an optical cavity with an injected squeezed vacuum," Phys. Rev. A 54, 5327-5333 (1996)

14. G. Tóth, C. Knapp, O. Gühne, and H. J. Briegel, "Optimal spin squeezing inequalities detect bound entanglement in spin models," Phys. Rev. Lett. 99, 250405 (2007).

15. J. Ma, X. Wang, C. Sun, and F. Nori, "Quantum spin squeezing," Phys. Rep. 509, 89-165(2011).

16. M. Cunha, J. Dunningham, and V. Vedral, "Entanglement in single-particle systems," Proc. R. Soc. A 463, 2277-2286 (2007).

17. S. Van Enk, "Single-particle entanglement," Phys. Rev. A 72, 64306 (2005).

18. F. Benatti, R. Floreanini, and U. Marzolino, "Entanglement and squeezing with identical particles: ultracold atom quantum metrology," J. Phys. B 44, 091001 (2011).

19. B. Öztop, M. Ö. Oktel, Ö. E. Müstecaphığlu, and L. You, "Quantum entanglement of spin-1 bosons with coupled ground states in optical lattices," J. Phys. B 42, 145505 (2009).

20. G. Tóth, C. Knapp, O. Gühne, and H. Briegel, "Spin squeezing and entanglement," Phys. Rev. A 79, 042334 (2009).

21. J. Vidal, "Concurrence in collective models," Phys. Rev. A 73, 062318 (2006)

22. G. Vitagliano, P. Hyllus, I. L. Egusquiza, and G. Tóth, "Spin squeezing inequalities for arbitrary spin," Phys. Rev. Lett. 107, 240502 (2011)

23. Y. Wu and X. Yang, "Effective two-level model for a three-level atom in the $\Xi$ configuration," Phys. Rev. A 56, 2443-2446 (1997).

24. Y. Wu, "Effective Raman theory for a three-level atom in the $\lambda$ configuration," Phys. Rev. A 54, 1586 (1996).

25. M. Alexanian and S. K. Bose, "Unitary transformation and the dynamics of a three-level atom interacting with two quantized field modes," Phys. Rev. A 52, 2218-2224 (1995).

26. M. Alexanian, S. Bose, and L. Chow, "Trapping and Fock state generation in a two-photon micromaser," J. Mod. Opt. 45, 2519-2532 (1998).
27. J. Vidal, G. Palacios, and C. Aslangul, "Entanglement dynamics in the Lipkin-Meshkov-Glick model," Phys. Rev. A 70, 062304 (2004).

28. P. Ribeiro, J. Vidal, and R. Mosseri, "Exact spectrum of the Lipkin-Meshkov-Glick model in the thermodynamic limit and finite-size corrections," Phys. Rev. E 78, 021106 (2008)

29. P. Ribeiro, J. Vidal, and R. Mosseri, "Thermodynamical limit of the Lipkin-Meshkov-Glick model," Phys. Rev. Lett. 99, 050402 (2007)

30. V. Karassiov and A. Klimov, "An algebraic approach to solving evolution problems in some nonlinear quantum models," Phys. Lett. A 189, 43-51 (1994).

31. P. Higgs, "Dynamical symmetries in a spherical geometry. I," J. Phys. A Math. Gen. 12, 309-323 (1979).

32. H. J. Carmichael, "Quantum fluctuations in absorptive bistability without adiabatic elimination,” Phys. Rev. A 33, 3262-3269 (1986).

33. M. Alexanian, "Scattering of two coherent photons inside a one-dimensional coupled-resonator waveguide," Phys. Rev. A 81, 015805 (2010).

34. J. Schreier, A. Houck, J. Koch, D. Schuster, B. Johnson, J. Chow, J. Gambetta, J. Majer, L. Frunzio, M. Devoret, S. M. Girvin, and R. J. Schoelkopf, "Suppressing charge noise decoherence in superconducting charge qubits," Phys. Rev. B 77, 180502 (2008)

35. C. Yang, "Fast quantum information transfer with superconducting flux qubits coupled to a cavity," J. Phys. A Math. Theor. 45 205304 (2012).

36. D. Petrosyan, G. Bensky, G. Kurizki, I. Mazets, J. Majer, and J. Schmiedmayer, "Reversible state transfer between superconducting qubits and atomic ensembles," Phys. Rev. A 79, 040304 (2009).

37. P. Forn-Díaz, J. Lisenfeld, D. Marcos, J. J. García-Ripoll, E. Solano, C. J. P. M. Harmans, and J. E. Mooij, "Observation of the Bloch-Siegert shift in a qubit-oscillator system in the ultrastrong coupling regime," Phys. Rev. Lett. 105, 237001 (2010).

38. J. Casanova, G. Romero, I. Lizuain, J. J. García-Ripoll, and E. Solano, "Deep strong coupling regime of the JaynesCummings model," Phys. Rev. Lett. 105, 263603 (2010).

39. M. Hofheinz, E. Weig, M. Ansmann, R. Bialczak, E. Lucero, M. Neeley, A. O'Connell, H. Wang, J. Martinis, and A. Cleland, "Generation of Fock states in a superconducting quantum circuit," Nature 454, 310-314 (2008).

40. D. Bozyigit, C. Lang, L. Steffen, J. Fink, C. Eichler, M. Baur, R. Bianchetti, P. Leek, S. Filipp, M. da Silva, A. Blais, and A. Wallraff, "Antibunching of microwave-frequency photons observed in correlation measurements using linear detectors," Nat. Phys. 7, 154-158 (2010). 